\title{
Evidence for a non-Rydberg molecular doubly excited state of $\mathrm{Ca}_{2}$
}

\author{
M.-A. Gaveau, J.-M. Mestdagh \\ T. Bouissou*, G. Durand*, M.-C Heitz*, F. Spiegelman* \\ Laboratoire Francis Perrin, \\ CEA/DSM/IRAMIS/SPAM - CNRS URA 2453, C.E.A., \\ Saclay, F-91191 Gif-sur-Yvette cedex, France \\ * Laboratoire de Physique et Chimie Quantiques, \\ CNRS UMR 5626, IRSAMC, \\ Université Paul Sabatier, 118 Route de Narbonne, \\ F31062 Toulouse cedex, France
}

Corresponding author: J.-M Mestdagh 


\begin{abstract}
We report experimental and theoretical evidence of a non-Rydberg molecular doubly excited state of $\mathrm{Ca}_{2}$ involving excited molecular orbitals mixing $4 p$ and $3 d$ characters. The excitation spectrum of $\mathrm{Ca}_{2}$, carried by helium or argon clusters, is recorded experimentally in the range $25600-27800 \mathrm{~cm}^{-1}$, displaying a bimodal structure. The latter is interpreted from $a b$ initio calculation and analysis of the adiabatic states of $\mathrm{Ca}_{2}$ up to doubly excited asymptotes $\mathrm{Ca}\left(4 \mathrm{p} 3 \mathrm{~d}^{1} \mathrm{D}\right)+\mathrm{Ca}\left(4 \mathrm{~s}^{2}{ }^{1} \mathrm{~S}\right)$ and $\mathrm{Ca}\left(4 \mathrm{~s} 3 \mathrm{~d}^{3} \mathrm{D}\right)+\mathrm{Ca}\left(4 \mathrm{~s} 4 \mathrm{p}^{3} \mathrm{P}\right)$, and the relevant dipole transition moments. The bimodal structure is assigned as resulting from the avoided crossing between adiabatic states $3{ }^{1} \Pi_{\mathrm{u}}$ and $4^{1} \Pi_{\mathrm{u}}$, reflecting the mixing of doubly excited configurations and absorbing singly excited configurations.
\end{abstract}




\section{Introduction}

An impressively large amount of spectroscopic data concern the photoexcitation and the photodissociation of diatomic molecules. This provides an important body of information on the electronic and spectral properties of molecules in addition to ab initio calculations which become more and more efficient. Cross-comparison constitutes a stringent way to interpret and assign the nature of the states, which is especially useful for high-lying states. Most experimental studies document states, whose main configuration is singly excited with respect to the ground state $[1,2]$. Besides of alkali dimers, which can be considered as two-electron systems and which can receive almost exact valence treatment, accurate theoretical prediction of the energy of doubly excited molecular states is still delicate in ab initio calculations. Getting accurate spectroscopic information on these states is therefore especially important, but difficult to obtain. Doubly excited states are indeed vanishingly coupled to the ground state by single photon transitions, hence making spectroscopic investigations difficult. A way to circumvent this difficulty is to take advantage of the coupling between a doubly excited state and spectroscopically accessible states. Spin-orbit coupling may sometimes provide favorable cases. For instance valence double excitations involving fine structure within a given molecular configuration have been reported for most halogen pairs, such as for instance the $\mathrm{I}^{*}\left({ }^{2} \mathrm{P}_{1 / 2}\right)-\mathrm{I}^{*}\left({ }^{2} \mathrm{P}_{1 / 2}\right)$ contact pair emission observed by Apkarian and coll. [3]. If we now consider actual electron configuration double excitations (not involving fine structure), a number of examples reported so far were concerned with quite large double excitation energies and the coupling used for spectroscopic purposes involved an autoionization channel. For instance, doubly excited states of molecules $\mathrm{H}_{2}, \mathrm{~N}_{2}$ and $\mathrm{CO}$ show up as resonances in their photoionization spectra $[4,5,6]$. In the context of ultracold collisions, doubly excited states of the $\mathrm{Na}_{2}$ molecule link the association reaction between two $\mathrm{Na}\left(3 \mathrm{p}^{2} \mathrm{P}\right)$ atoms to the associative ionization forming $\mathrm{Na}_{2}^{+}[7,8]$.

A very different situation exists with metal atom dimers when the metal has an 
energetically low lying, high spin state, whose energy is less than half the ionization energy. When associating two such metal atoms, one (or several) bonded doubly excited state(s) may exist, whose energy is lower than that of the molecular ion. This situation is expected to be quite common with transition metals and lanthanides $[9,10,11]$, often characterized as mixed valence compounds especially challenging from the electronic structure point of view and their magnetic properties. A similar situation also occurs in alkaline-earth metals. In such case, investigations using the above mentioned spectroscopic studies are not possible, since no autoionization channel is opened. Such doubly excited states are spectroscopically almost "invisible" and can be observed only through configuration mixing with singly excited states of same symmetry, acquiring non-vanishing dipole transition moments. This type of situation has been predicted theoretically for $\mathrm{Ba}_{2}$, but not yet observed [12].

The present work is both experimental and theoretical. It reports the investigation of low lying doubly excited states, taking another alkaline earth dimer, $\mathrm{Ca}_{2}$, as an example. Calcium is very favorable for this purpose. The energy of its lower triplet state, $\mathrm{Ca}\left(4 \mathrm{~s} 4 \mathrm{p}^{3} \mathrm{P}\right)$, is $15263 \mathrm{~cm}^{-1}$, whereas the ionization energy of $\mathrm{Ca}$ is more than a factor 3 larger $\left(49306 \mathrm{~cm}^{-1}\right)$. The next triplet state, $\mathrm{Ca}\left(4 \mathrm{~s} 3 \mathrm{~d}^{3} \mathrm{D}\right)$ lies at quite low energy also $\left(20357 \mathrm{~cm}^{-1}\right)$ [9]. Doubly excited states of $\mathrm{Ca}_{2}$ correlating asymptotically with $\mathrm{Ca}\left(4 \mathrm{~s} 4 \mathrm{p}{ }^{3} \mathrm{P}\right)+\mathrm{Ca}\left(4 \mathrm{~s} 4 \mathrm{p}^{3} \mathrm{P}\right)$ or with $\mathrm{Ca}\left(4 \mathrm{~s} 4 \mathrm{p}{ }^{3} \mathrm{P}\right)+\mathrm{Ca}\left(4 \mathrm{~s} 3 \mathrm{~d}^{3} \mathrm{D}\right)$ are thus expected to show interactions in the short range region with states correlating asymptotically with the singly excited neutral pair at $23652 \mathrm{~cm}^{-1}, \mathrm{Ca}\left(4 \mathrm{~s} 4 \mathrm{p}{ }^{1} \mathrm{P}\right)+\mathrm{Ca}\left(4 \mathrm{~s}^{2}{ }^{1} \mathrm{~S}\right)$.

Besides of the intrinsic motivation for investigating doubly excited electronic states, an accurate knowledge of the spectroscopy of $\mathrm{Ca}_{2}$ is of interest since the $\mathrm{Ca}\left(4 \mathrm{~s} 4 \mathrm{p}{ }^{1} \mathrm{P}\right)+$ $\mathrm{Ca}\left(4 \mathrm{~s}^{2}{ }^{1} \mathrm{~S}\right)$ collision has been proposed as a likely candidate for a photoassociation reaction in order to generate ultracold $\mathrm{Ca}_{2}$ molecules $[13,14,15]$.

The present experimental investigation of the calcium dimer spectroscopy is performed using a setup allowing for generation of molecules trapped both on argon clusters and on helium droplets. Such a setup can be used to investigate molecule-cluster 
interaction and cluster properties, but it is also a practical tool for molecular spectroscopy itself, especially when used with helium droplets, which is a goal in the present work. The pick up technique indeed favors molecule formation and even clustering on the cold, inert host cluster. It is complementary to isolated molecules studies. The set up is presented in section 2, together with the excitation spectrum in the range 25600-27800 $\mathrm{cm}^{-1}$. The relevant potential curves and dipole moments are calculated in section 3. The interpretation of the experimental data via the calculated absorption spectrum is given in section 4 .

\section{Experimental : set up and excitation spectrum in the region $25600-27800 \mathrm{~cm}^{-1}$}

The $\mathrm{Ca}_{2}$ molecules are carried either by argon clusters or by helium droplets, using the Cluster Isolated Chemical Reaction technique $[16,17]$. We chose this technique because of its flexibility, although direct supersonic expansion could be used to generate the $\mathrm{Ca}_{2}$ molecules. The experiments are performed in the same spirit as those on the reaction dynamics of barium clusters [18] and on the spectroscopy of low lying electronic states of $\mathrm{Ca}_{2}$ dimers [19]. Their principle is resolved in five steps: i) large argon clusters or helium droplets are generated in a supersonic expansion; ii) a controlled average number $\overline{\mathrm{N}}$ of calcium atoms is deposited per cluster using the collisional pickup technique; iii) given the low temperature of the clusters $(\mathrm{T}=0.4 \mathrm{~K}$ for helium droplets [20], $32 \mathrm{~K}$ for argon clusters [21]), the calcium atoms associate together and form an aggregate; iv) the desired process, here the photodissociation of $\mathrm{Ca}_{2}$ producing

the luminescent $\mathrm{Ca}\left(4 \mathrm{~s} 4 \mathrm{p}^{1} \mathrm{P}\right)$ atom, is induced; $\left.v\right)$ the light emission of $\mathrm{Ca}\left(4 \mathrm{~s} 4 \mathrm{p}^{1} \mathrm{P}\right)$ is studied as a function of the wavelength of the laser that photodissociates $\mathrm{Ca}_{2}$, hence providing the action spectrum of the desired photodissociation process

$$
\mathrm{Ca}_{2} \stackrel{h \nu}{\longrightarrow} \mathrm{Ca}\left(4 \mathrm{~s}^{2}{ }^{1} \mathrm{~S}\right)+\mathrm{Ca}\left(4 \mathrm{~s} 4 \mathrm{p}{ }^{1} \mathrm{P}\right) .
$$

The argon clusters are produced in a molecular beam source of the Camparguetype [22]. The supersonic expansion proceeds from a 20 bar stagnation pressure at 
room temperature through a $0.2 \mathrm{~mm}$ nozzle. It generates clusters with an average size of $\approx 2000$ [23]. The source used to generate the helium droplets is a modified Campargue-type supersonic beam $[22,24]$. Helium with a stagnation pressure of 7.5 bars is expanded through a $0.005 \mathrm{~mm}$ nozzle that is refrigerated down to $10 \mathrm{~K}$. The size of the clusters is estimated to a few thousands [25]. After a double extraction by a $1 \mathrm{~mm}$ skimmer and a $3 \mathrm{~mm}$ collimator, the cluster beam enters the pick-up zone, a $30 \mathrm{~mm}$ long heated cell $\left(\mathrm{T} \simeq 520^{\circ} \mathrm{C}\right)$, where the calcium atoms are captured. Varying the cell temperature allows the calcium vapor pressure to be adjusted, hence making the average number of $\mathrm{Ca}$ atoms deposited per cluster adjustable [16]. Afterwards, the beam crosses a region where both laser excitation and fluorescence detection are performed. The beam is illuminated there by the light of a $c w$ Titanium:Sapphire laser doubled by an extra cavity doubler. The fluorescence light that is emitted at the same place is collected, dispersed by a scanning grating monochromator and detected by a cooled photomultiplier tube using the photon counting technique.

The laser is tuned in the range $360-390 \mathrm{~nm}\left(25640-27780 \mathrm{~cm}^{-1}\right)$ resulting in a calcium emission that is recorded as a function of the laser wavelength. This emission cannot be due to a laser induced fluorescence signal of atomic calcium since the latter has no dipole-allowed line in this energy region. Indeed, beyond the atomic resonant line to $4 \mathrm{~s} 4 \mathrm{p}^{1} \mathrm{P}$ at $23652 \mathrm{~cm}^{-1}$, the closest atomic levels [9] lie $4 \mathrm{~s} 5 \mathrm{~s}^{3,1} \mathrm{~S}$ at 31539 and $33000 \mathrm{~cm}^{-1}$, both dipole-forbidden, and the next resonant line towards $4 \mathrm{~s} 5 \mathrm{P}^{1} \mathrm{P}$ lies at $37242 \mathrm{~cm}^{-1}$. Neither the helium droplet, nor the argon clusters are believed to exert such a large shift on the calcium levels. Hence, the observed emission is not caused by the absorption of the laser light by atomic calcium. Instead, we shall see below that it results from the absorption by the $\mathrm{Ca}_{2}$ molecule followed by process 1 .

Two excitation spectra of this fluorescence are shown in Fig. 1. They correspond to the helium (solid curve) and the argon (dashed curve) experiments. They are very similar. An intense band is observed, close to a blue shifted side band. The main band of the helium experiment peaks at $26495 \pm 10 \mathrm{~cm}^{-1}$. The corresponding FWHM (Full 
Width at Half the Maximum) is $300 \mathrm{~cm}^{-1}$. The side band is blue shifted by $550 \mathrm{~cm}^{-1}$ with respect to the main band and the FWHM is $50 \mathrm{~cm}^{-1}$ wider. The corresponding bands in the argon experiment are red shifted by c.a. $150 \mathrm{~cm}^{-1}$ compared to the helium experiment.

Two methods allow us to check that the fluorescence emission follows the absorption of the laser light by a $\mathrm{Ca}_{2}$ molecule. First, the fluorescence intensity can be explored as a function of $\overline{\mathrm{N}}$, the average number of Ca atoms per cluster. Taking advantage that the Poisson distribution describes the statistics of the collisional pick up, the observed signal is related to a specific order of the Poisson laws, thus telling the exact number $\mathrm{n}$ of reactants responsible for the observed signal [16]. This was done in the argon cluster experiment. The total fluorescence intensity is plotted in Fig. 2 as a function of $\bar{N}$. As observed in the figure, the fluorescence signal follows the second order Poisson law, indicating that it is produced by clusters carrying two and only two calcium atoms. On a cold cluster ( $\mathrm{T}=0.4 \mathrm{~K}$ for helium droplets [20], $32 \mathrm{~K}$ for argon clusters [21]) of finite size, such two atoms are likely to meet and combine rapidly. Hence the emission results from the excitation of a $\mathrm{Ca}_{2}$ molecule, that has been stabilized on the cluster after the successive deposition of two calcium atoms. Alternatively, since only information on the dimer is desired, it is sufficient to adjust the Calcium cell temperature to keep $\overline{\mathrm{N}}$ at a small enough value insuring that measured signals cannot be due to larger clusters [16]. This was done in the helium droplet experiment.

The observed emission was dispersed using the monochromator. It corresponds to the $\mathrm{Ca}\left(4 \mathrm{~s} 4 \mathrm{p}^{1} \mathrm{P} \rightarrow 4 \mathrm{~s}^{2} \mathrm{~S}\right)$ emission. Hence, the spectra shown in Fig. 1 are the action spectra of process 1 when the $\mathrm{Ca}_{2}$ molecule is carried either by a helium droplet or an argon cluster.

In the helium droplet experiment, the observed emission corresponds to the $\mathrm{Ca}\left(4 \mathrm{~s} 4 \mathrm{p}{ }^{1} \mathrm{P} \rightarrow\right.$ $4 \mathrm{~s}^{2} \mathrm{~S}$ ) transition of free calcium with no side band. This indicates that the photodissociation of $\mathrm{Ca}_{2}$ ejects the electronically excited Ca atom off the droplet, with a $100 \%$ probability. In contrast, a sidebands exist in the argon experiment. It is located to the 
red of the calcium resonance line, an indication that part of the departing $\mathrm{Ca}\left(4 \mathrm{~s} 4 \mathrm{p}{ }^{1} \mathrm{P}\right)$ atoms stay solvated on the cluster. Hence, the desorption dynamics of $\mathrm{Ca}\left(4 \mathrm{~s} 4 \mathrm{p}^{1} \mathrm{P}\right)$ is different whether it interacts with an helium droplet or an argon cluster. This question, is beyond the scope of the present work.

\section{Theory: ${ }^{1} \Sigma_{u}^{+},{ }^{1} \Pi_{u}$ potential energy curves and transition dipole moments}

In order to rationalize the absorption features recorded experimentally, molecular states dissociating as high as the $\mathrm{Ca}\left(4 \mathrm{~s} 3 \mathrm{~d}^{3} \mathrm{D}\right)+\mathrm{Ca}\left(4 \mathrm{~s} 4 \mathrm{p}{ }^{3} \mathrm{P}\right)$ and $\mathrm{Ca}\left(4 \mathrm{p} 3 \mathrm{~d}^{1} \mathrm{D}\right)+\mathrm{Ca}\left(4 \mathrm{~s}^{2}{ }^{1} \mathrm{~S}\right)$ asymptotical limits were calculated. This corresponds to a wider electronic spectral range than available in previous calculations [26, 27], and was necessary, due to the attractive character of some of those double excited states, as will be seen below. The relevant electronic spectrum of $\mathrm{Ca}_{2}$ is investigated using ab initio calculations where the $\mathrm{Ca}_{2}$ molecule is reduced to four active electrons via effective core pseudopotentials and core-polarisation pseudopotentials [28] (CPP). The excited states were obtained through the hybrid variational perturbative multi-reference MRPT2/configuration interaction scheme CIPSI [29] in its quasi-degenerate perturbation theory version, in order to treat consistently perturbative contributions in the vicinity of avoided crossings [30]. A 8s8p8d4f gaussian basis set has been used on each calcium atom. The average error on atomic transitions was found to be of the order of $250 \mathrm{~cm}^{-1}$, with a maximal error of $\sim 450 \mathrm{~cm}^{-1}$. The present results for the lowest molecular states of $\mathrm{Ca}_{2}$, dissociating up to $\mathrm{Ca}\left(4 \mathrm{~s} 4 \mathrm{P}^{1} \mathrm{D}\right)+\mathrm{Ca}\left(4 \mathrm{~s}^{2}\right)$ are in general agreement with previous calculations [27], in particular with those of Czuchaj et al. [26] realized with a similar methodology. A detailed discussion of the calculated potential curves for all symmetry manifolds will be given in a forthcoming publication [31].

Both ${ }^{1} \Sigma_{u}^{+}$and ${ }^{1} \Pi_{\mathrm{u}}$ states are dipole-allowed from the ground state. Fig. 3 shows the ${ }^{1} \Sigma_{\mathrm{u}}^{+}$and and ${ }^{1} \Pi_{\mathrm{u}}$ potential energy curves in the energy range $20000-30000 \mathrm{~cm}^{-1}$. The vertical dashed line shows the Ca-Ca equilibrium distance in the ground state molecule, 
$\mathrm{R}_{\mathrm{e}}=8.1 \mathrm{a}_{0}$. At that distance, states $2^{1} \Sigma_{\mathrm{u}}^{+}$and $3^{1} \Sigma_{\mathrm{u}}^{+}$lie at 19673 and $27970 \mathrm{~cm}^{-1}$ above the ground state. The latter state, dissociating into $\mathrm{Ca}\left(4 \mathrm{~s}^{2}{ }^{1} \mathrm{~S}\right)+\mathrm{Ca}\left(4 \mathrm{~s} 5 \mathrm{~s}^{1} \mathrm{~S}\right)$, has a transition shifted significantly to the blue of the experimental action spectrum. Moreover, its calculated dipole transition moment from the ground state remains weak in the range $\mathrm{R}>5 \mathrm{a}_{0}\left(\mu=0.33\right.$ a.u. at $\left.\mathrm{R}=8.1 \mathrm{a}_{0}\right)$, much less than that corresponding to the $2-3^{1} \Pi_{\mathrm{u}}$ states close to the avoided crossing ( $\mu \approx 2$ a.u, see below), which results in a factor $1 / 30$ in the intensities with respect to the main absorption of the latter states. Considering those data, we think that the ${ }^{1} \Pi_{u}$ states only are responsable for the present absorption, and we focus the discussion on them.

The three adiabatic states 2,3 and $4^{1} \Pi_{\mathrm{u}}$ in the region between 20000 and $27000 \mathrm{~cm}^{-1}$ are directly relevant to analyze the experimental results of Fig. 1. They are discussed below as resulting from multiple avoided crossings between mainly three diabatic components. A first diabatic state is correlated with the singly excited asymptote $\mathrm{Ca}\left(4 \mathrm{~s} 4 \mathrm{p}{ }^{1} \mathrm{P}\right)+\mathrm{Ca}\left(4 \mathrm{~s}^{2} \mathrm{~S}\right)$. It corresponds to the adiabatic state $2^{1} \Pi_{\mathrm{u}}$ for $\mathrm{R}>10 \mathrm{a}_{0}$. It is repulsive, and contributes to two avoided crossings, the first one at $R \approx 10 \mathrm{a}_{0}$, the second one at $\mathrm{R} \approx 7.9 \mathrm{a}_{0}$. Inspection of the electronic wavefunctions reveals that asymptotically, it is spanned by a mixing of $\sigma_{\mathrm{u}}(4 \mathrm{~s}) \rightarrow \pi_{\mathrm{g}}(4 \mathrm{p})$ and $\sigma_{\mathrm{g}}(4 \mathrm{~s}) \rightarrow \pi_{\mathrm{u}}(4 \mathrm{p})$ configurations with equal weights. At shorter distance however, the weights of configurations with a hole in $\sigma_{\mathrm{g}}(4 \mathrm{~s})$ decrease, while both $4 \mathrm{p}$ and $3 \mathrm{~d}$ characters contribute to the two first excited molecular orbitals in each symmetry, due to the lower lying ${ }^{1} \mathrm{D}$ atomic asymptote. A second diabatic state correlates with the doubly excited $\mathrm{Ca}\left(4 \mathrm{~s} 4 \mathrm{p}^{3} \mathrm{P}\right)+\mathrm{Ca}\left(4 \mathrm{~s} 4 \mathrm{p}^{3} \mathrm{P}\right)$ asymptote. It generates two avoided crossings, the one at $\mathrm{R} \approx 10 \mathrm{a}_{0}$ already mentioned and one at $\mathrm{R} \approx 7 \mathrm{a}_{0}$, with a third diabatic state, characterized by a stronger and less localized coupling. The latter state has 4p3d doubly excited character. Asymptotically, the two states $\mathrm{Ca}\left(4 \mathrm{~s} 4 \mathrm{p}^{3} \mathrm{P}\right)+\mathrm{Ca}\left(4 \mathrm{~s} 3 \mathrm{~d}^{3} \mathrm{D}\right)$ (having simultaneous single excitations on both atoms) and $\mathrm{Ca}\left(4 \mathrm{~s}^{2}{ }^{1} \mathrm{~S}\right)+\mathrm{Ca}\left(4 \mathrm{p} 3 \mathrm{~d}{ }^{1} \mathrm{D}\right)$ (with a double excitation on a single atom) are almost degenerate (experimentally $\left.\Delta \mathrm{E} \approx 200 \mathrm{~cm}^{-1}\right)$. For distances less than $\mathrm{R}=15 \mathrm{a}_{0}$ both mix and contribute into a 
strongly bound diabatic state essentially spanned by double excitation $\sigma_{\mathrm{u}}(4 \mathrm{~s})^{2} \rightarrow \sigma_{\mathrm{g}} \pi_{\mathrm{u}}$ where both $\sigma_{\mathrm{g}}$ and $\pi_{\mathrm{u}}$ orbitals mix $4 \mathrm{p}$ and $3 \mathrm{~d}$ characters. This strongly bonding contribution, due to emptying the antibonding $\sigma_{\mathrm{u}}(4 \mathrm{~s})$ orbital, causes this diabatic state to undergo two crossings with lower states, and finally results into the short range minimum of adiabatic state $2^{1} \Pi_{\mathrm{u}}$ at $\mathrm{R}=5.96 \mathrm{a}_{0}$ and $\mathrm{T}_{\mathrm{e}}=20852 \mathrm{~cm}^{-1}$. The above analysis results from the examination of the dominant configurations in the adiabatic electronic wavefunction. The vicinity of the avoided crossing at $\mathrm{R}=7.9 \mathrm{a}_{0}$ is a zone of special interest for the comparison with the experimental results. At $R=7.5 \mathrm{a}_{0}$, the adiabatic states $3^{1} \Pi_{\mathrm{u}}$ and $4^{1} \Pi_{\mathrm{u}}$ are dominated by configurations $\sigma_{\mathrm{u}}^{2} \rightarrow \sigma_{\mathrm{g}} \pi_{\mathrm{u}}$ and $\sigma_{\mathrm{u}} \rightarrow \pi_{\mathrm{g}}(4 \mathrm{p})$ respectively, whereas at $\mathrm{R}=8.5 \mathrm{a}_{0}$, the characters are switched into one another.

The nature of the excited states is globally rationalized in the previous discussion as two doubly excited diabatic states (strongly bonding), interacting with a singly excited diabatic state (repulsive) and thus yielding directly to the dissociation monitored experimentally. The configuration mixing discussed above is illustrated further by calculating the electric dipole transition moments from the ground state. It is shown in Fig. 4. With decreasing distance, the dipole transition moment of the absorbing state $2^{1} \Pi_{u}$ correlated with the atomic resonance line, switches with that of $3^{1} \Pi_{u}$ and again with that of state $4^{1} \Pi_{\mathrm{u}}$. This variation of the transition dipole moments influences certainly the shape of the absorption spectrum of the $3{ }^{1} \Pi_{u}$ and $4{ }^{1} \Pi_{u}$ states, allowing for a comparison with the experimental spectrum.

\section{Discussion}

The ressemblance between the two action spectra, shown in Fig. 1 respectively for helium and argon droplets, is striking. This seems surprising given the $\mathrm{Ca} / \mathrm{Ar}$ and $\mathrm{Ca} / \mathrm{He}$ interactions are different $[32,33]$. This must be related to the fact that, likely, the molecule is carried at the surface of either the argon cluster or the helium droplet. In former theoretical and spectroscopic investigations, we have shown that a single 
$\mathrm{Ca}$ atom is located at the surface of the argon cluster $[19,34]$, linked to the smaller dissociation energy of $\mathrm{Ca}-\mathrm{Ar}$ and larger equilibrium distance with respect to Ar-Ar. In the case of helium droplets, early laser induced fluorescence of atomic calcium, in the vicinity of the $\mathrm{Ca}\left(4 \mathrm{~s} 4 \mathrm{p}^{1} \mathrm{P} \leftarrow 4 \mathrm{~s}^{2}{ }^{1} \mathrm{~S}\right)$ resonance line, suggested a surface location for the calcium atom [35]. This was confirmed later by photoionization spectroscopy [36]. In helium droplets, quantum vibration dynamics must be considered in addition to comparison of the interactions. Recent calculations [37] using vibrational time-dependent density functional theory with additive potentials built from high quality ground and excited $\mathrm{CaHe}$ interactions, also confirmed the location of $\mathrm{Ca}$ in surface dimples, and were able to reproduce the magnitudes of the shifts and widths in the resonant absorption line of Ca observed by Stienkemeier et al. $\mathrm{Ca}_{2}$ is essentially a Van der Waals molecule, despite of its rather large bonding $\left(D_{\mathrm{e}}=1016 \mathrm{~cm}^{-1}\right)$. One may similarly expect the calcium molecule to be located at the surface of the droplet.

The theoretical absorption spectrum for states $3{ }^{1} \Pi_{u}$ and $4^{1} \Pi_{u}$ was determined as the Fourier transform of the autocorrelation function calculated by wavepacket propagation on the two adiabatic potential energy curves (see ref. [38]). The vibrational structure of the spectrum has been washed out by applying an exponential damping function on the autocorrelation function in order to take into account in a phenomenological way both the electronic nonadiabatic couplings between the two states and the vibrational coupling of the diatomic molecule with the cluster. The theoretical absorption spectrum is shown in Fig. 5 (shifted by $450 \mathrm{~cm}^{-1}$ to the blue) for comparison with experimental data on helium droplets and in Fig. 3 in superimposition to the potential energy curves. The calculated absorption spectrum exhibits a bimodal structure that directly results from the avoided crossing between the singly and doubly excited states, close to the Franck-Condon region. In particular, the blue side of the experimentally recorded spectra can be assigned to the observation of a non-Rydberg doubly excited state, mediated by the coupling with a strongly absorbing singly excited state. This derives from the discussion above and is well illustrated in Fig. 3. 
Fig. 5 shows that both the theoretical absorption spectrum and the experimental action spectrum for helium (where the interaction with the cluster is expected to be the weakest) have very similar bimodal shapes. The theoretical width of the intense red component is in excellent agreement with the experiment. Moreover, the oscillator strength of the transitions around wavenumber $26500 \mathrm{~cm}^{-1}$ is found to be larger than that of the blue maximum: a factor 12.5 is found in the experimental signal, a factor 6 in the calculation. Obviously, and since the anticrossing occurs at the vertical of the initial ground state, this can be sensitive to the quality of the calculation and the accurate position of the avoided crossing above the ground state. Here, we have compared the action spectrum of the photodissociation process 1 on helium measured experimentally and the absorption spectrum of the $\mathrm{Ca}_{2}$ molecule simulated theoretically. This comparison is relevant only if exciting the molecule to either state, $3^{1} \Pi_{u}$ or $4^{1} \Pi_{u}$, close to the anticrossing at $\mathrm{R}=7.9 \mathrm{a}_{0}$ actually fully yields to the $\mathrm{Ca}\left(4 \mathrm{~s}^{2}{ }^{1} \mathrm{~S}\right)+\mathrm{Ca}\left(4 \mathrm{~s} 4 \mathrm{p}{ }^{1} \mathrm{P}\right)$ dissociation. The potential curves shown in Fig. 3 suggest that this should be approximately the case. The two anticrossings experienced by state $3{ }^{1} \Pi_{u}$ with state $2^{1} \Pi_{u}$ are not likely to significantly quench the dissociation. Nevertheless, it is not excluded that some flux is funneled to the lower molecular states $2^{1} \Pi_{u}$ and $1^{1} \Pi_{u}$. Dissociation quenching could also be enhanced by the presence of the helium droplet or the argon cluster. Neglect of those processes might be an explanation for intensity discrepancies of the blue sideband in the experimental spectrum, as compared to that of the calculated spectrum. Non-adiabatic dynamical calculations including all the states and the interaction with the rare gas, which are beyond the scope of the present letter, should help to confirm this hypothesis. Work is in progress towards this goal.

The present investigation shows that doubly excited states play an essential role in the spectroscopy of $\mathrm{Ca}_{2}$ in the energy range of 19000 to $27000 \mathrm{~cm}^{-1}$. The spectrum is complicated by the occurrence of several types of doubly excited molecular states, either correlated with pairs of simultaneously singly excited atoms, or with one ground state atom and another one in a doubly excited state. 
Importantly, helium clusters can also be used to accommodate reactive molecules in addition to $\mathrm{Ca}_{2}$ molecules. The kind of electronic excitation that is performed in the present work would enable to study the reaction dynamics of non Rydberg doubly excited states of $\mathrm{Ca}_{2}$ in a very direct way. To our knowledge, no such information is available yet, although investigation of reactivity within helium droplets is developing quickly $[39,40]$.

\section{Acknowledgments}

Supports from the CNRS-GDR 2758 "Agrégation, Fragmentation,Thermodynamique de Systèmes Complexes Isolés", the computational facilities of CALMIP/CICT Toulouse, the ANR-06-BLAN-031401 GOUTTELIUM and the ANR-07-BLAN-0156 DYNAMIX are acknowledged.

\section{References}

[1] W. Demtroder and I. Inguscio, editors, Applied laser spectroscopy, volume 241 of NATO ASI series : Series B, Plenum Pr., New York, 1990.

[2] D. L. Andrews and A. A. Demidov, editors, An introduction to laser spectroscopy, Laser spectroscopy, Springer, New York, 2nd edition, 2002.

[3] M. Karavitis and V. A. Apkarian, J. Phys. Chem. B 106, 8466 (2002).

[4] I. Sanchez and F. Martin, J. Chem. Phys. 106, 7720 (1997).

[5] P. Erman, A. Karawajczyk, U. Köble, E. Rachlew, K. Y. Franzén, and L. Veseth, Phys. Rev. Lett. 76, 4136 (1996).

[6] P. Erman, A. Karawajczyk, E. Rachlew-Källne, M. Stankiewicz, K. Y. Franzén, P. Sannes, and L. Veseth, Phys. Rev. A 55, 4221 (1997).

[7] X. Dai, H. Chen, Y. Liu, J. Li, J. Xiang, D. Chen, L. Li, and G. H. Jeung, J. Mol. Spectrosc. 198, 239 (1999). 
[8] A. Amelink, K. M. Jones, P. D. Lett, P. van der Straten, and H. G. M. Heideman, Phys. Rev. A 61, 042707 (2000).

[9] C. E Moore, Atomic Energy Levels, Circular 467, Vol I, NBS, US Dept. of Commerce, 1949.

[10] C. E Moore, Atomic Energy Levels, Circular 467, Vol II, NBS, US Dept. of Commerce, 1952.

[11] C. E Moore, Atomic Energy Levels, Circular 467, Vol III, NBS, US Dept. of Commerce, 1958.

[12] A. R. Allouche, M. Aubert-Frécon, G. Nicolas, and F. Spiegelmann, Chem. Phys. 200, 63 (1995).

[13] M. Machholm, P. S. Julienne, and K. A. Suominen, Phys. Rev. A 64, 033425 (2001).

[14] R. Ciurylo, E. Tiesinga, S. Kotochigova, and P. S. Julienne, Phys. Rev. A 70, $062710(2004)$.

[15] B. Bussery-Honvault, J. M. Launay, and R. Moszynski, Phys. Rev. A 68, 032718 (2003).

[16] J. M. Mestdagh, M. A. Gaveau, C. Gée, O. Sublemontier, and J. P. Visticot, Int. Rev. Phys. Chem. 16, 215 (1997).

[17] M. A. Gaveau, C. Gée, J. M. Mestdagh, and J. P. Visticot, Comments At. Mol. Phys. 34, 241 (1999).

[18] C. Gée, M. A. Gaveau, O. Sublemontier, J. M. Mestdagh, and J. P. Visticot, J. Chem. Phys. 107, 4194 (1997).

[19] M. A. Gaveau, M. Briant, P. R. Fournier, J. M. Mestdagh, and J. P. Visticot, J. Chem. Phys. 116, 955 (2002).

[20] M. Hartmann, R. E. Miller, J. P. Toennies, and A. Vilesov, Phys. Rev. Lett. 75, $1566(1995)$. 
[21] J. Farges, M. F. de Feraudy, B. Raoult, and G. Torchet, J. Chem. Phys. 84, 3491 (1986).

[22] R. Campargue, J. Phys. Chem. 88, 4466 (1984).

[23] J. Cuvellier, P. Meynadier, P. de Pujo, O. Sublemontier, J. P. Visticot, J. Berlande, A. Lallement, and J. M. Mestdagh, Z. Phys. D 21, 265 (1991).

[24] M.-A. Gaveau and P.-R. Fournier, A Helium Cluster Beam Source for Cluster Isolated Chemical Reactions, in XXVth International Symposium on Rarefied Gas Dynamics, edited by A. K. Rebrov and M. S. Ivanov, pages 1288-1295, St Petersbourg, Russia, 2007, Siberian Branch of the Russian Academy of Sciences: St Petersbourg (Russia).

[25] F. Stienkemeier and K. K. Lehmann, J. Phys. B-At. Mol. Opt. Phys. 39, R127 (2006).

[26] E. Czuchaj, M. Krosnicki, and H. Stoll, Theor. Chem. Acc. 110, 28 (2003).

[27] B. Bussery-Honvault and R. Moszynski, Mol. Phys. 104, 2387 (2006).

[28] W. Müller, J. Flesch, and W. Meyer, J. Chem. Phys. 80, 3297 (1984).

[29] B. Huron, J. P. Malrieu, and P. Rancurel, J. Chem. Phys. 58, 5745 (1973).

[30] F. Spiegelmann and J. P. Malrieu, J. Phys. B-At. Mol. Opt. Phys. 17, 1235 (1984).

[31] T. Bouissou, G. Durand, M.-C. Heitz, and F. Spiegelman, to be published.

[32] F. Spiegelman, L. Maron, W. H. Breckenridge, J. M. Mestdagh, and J. P. Visticot, J. Chem. Phys. 117, 7534 (2002).

[33] E. Czuchaj, F. Rebentrost, H. Stoll, and H. Preuss, Chem. Phys. Lett. 182, 191 (1991).

[34] M. A. Gaveau, M. Briant, P. R. Fournier, J. M. Mestdagh, J. P. Visticot, F. Calvo, S. Baudrand, and F. Spiegelman, Eur. Phys. J. D 21, 153 (2002).

[35] F. Stienkemeier, F. Meier, and H. O. Lutz, J. Chem. Phys. 107, 10816 (1997).

[36] Y. Ren and V. V. Kresin, Phys. Rev. A 76 (2007). 
[37] A. Hernando, M. Barranco, R. Mayol, M. Pi, and M. Krośinscky, Physical Review B 77 (2008).

[38] R. Schinke, Photodissociation Dynamics, Cambridge University Press, Cambridge, 1995.

[39] S. Denifl, F. Zappa, I. Mähr, J. Lecointre, T. D. Probst, M. Märk, and P. Scheier, Phys. Rev. Lett. 97, 043201 (2006).

[40] F. Zappa, S. Denifl, I. Mähr, A. Bacher, O. Echt, T. D. Märk, and P. Scheier, J. Am. Chem. Soc. 130, 5573 (2008). 


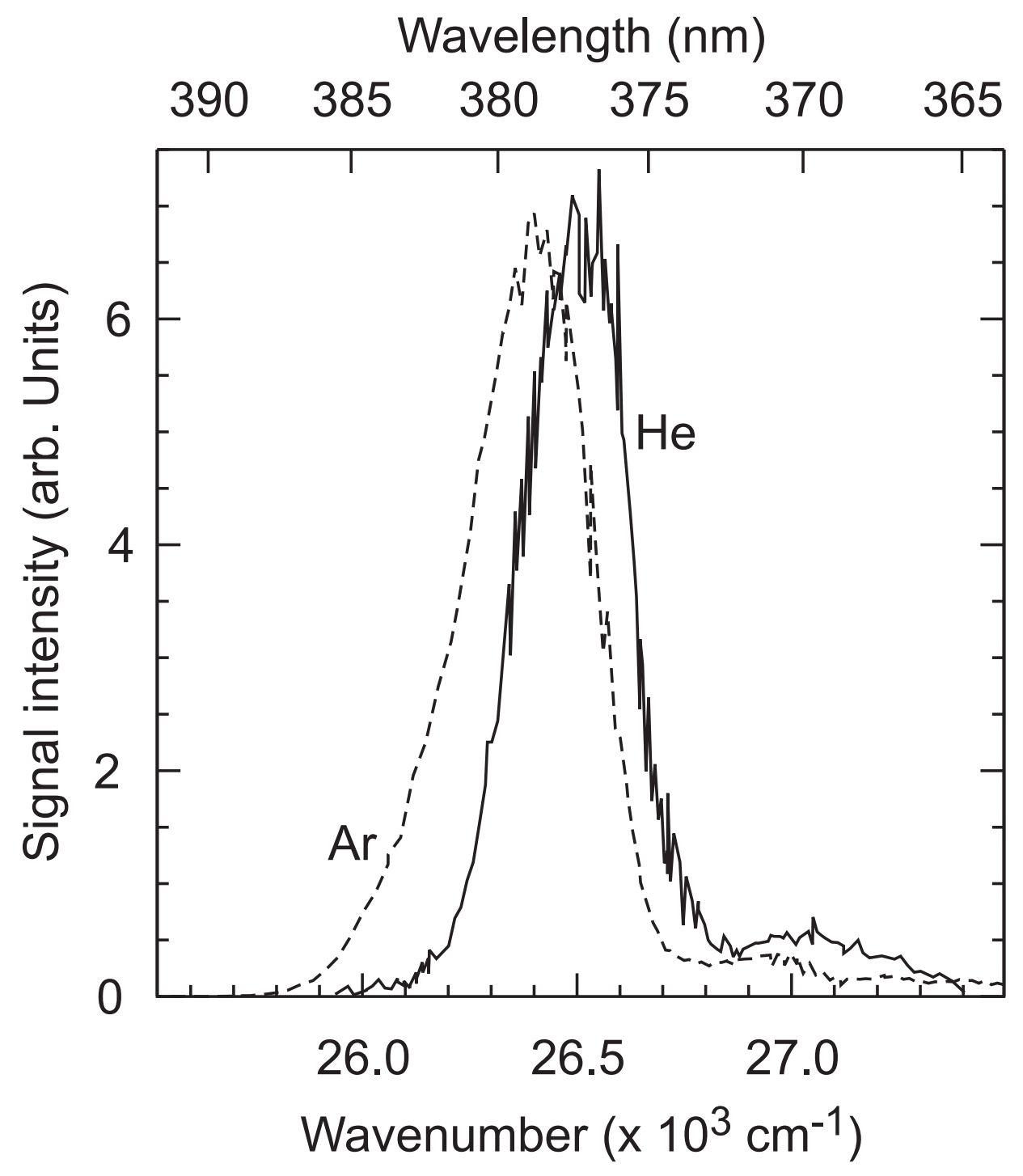

Figure 1: Excitation spectrum of the $\mathrm{Ca}\left(4 \mathrm{~s}^{2}{ }^{1} \mathrm{~S} \leftarrow 4 \mathrm{~s} 4 \mathrm{p}^{1} \mathrm{P}\right)$ fluorescence, i.e. action spectrum of process (1) when $\mathrm{Ca}_{2}$ is carried on helium droplets (solid line) and argon clusters (dashed line). The two spectra are normalized with respect to their intensity. 


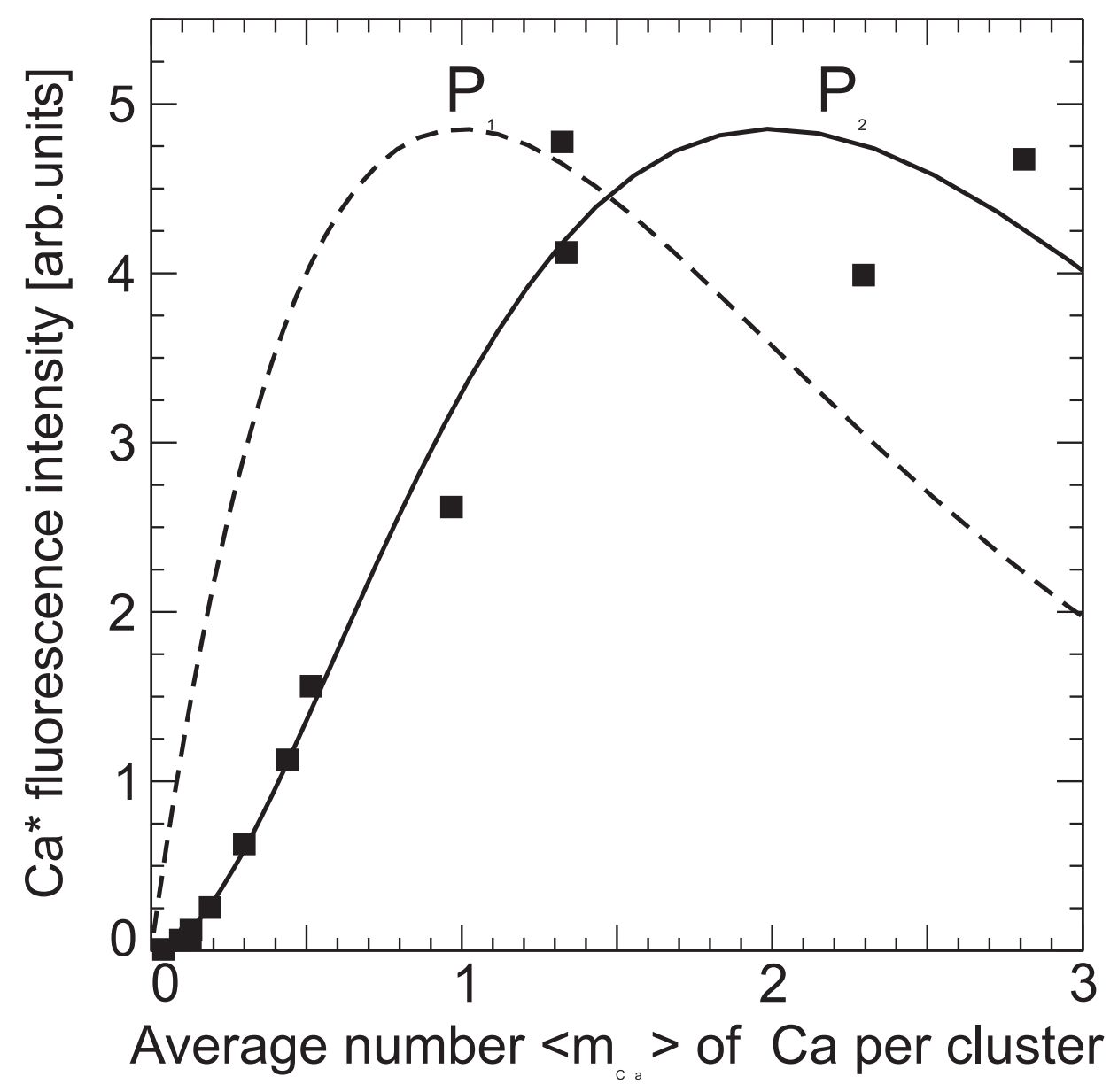

Figure 2: Evolution of the $\mathrm{Ca}\left(4 \mathrm{~s}^{2}{ }^{1} \mathrm{~S} \leftarrow 4 \mathrm{~s} 4 \mathrm{p}{ }^{1} \mathrm{P}\right)$ fluorescence emission as a function of the average number of Ca atoms per argon cluster (full squares). The excitation laser was tuned to $380 \mathrm{~nm}$. The curves labeled $\mathrm{P}_{1}$ and $\mathrm{P}_{2}$ refer to the Poisson laws of first and second order, respectively. They are normalized with respect to the maximum intensity of the experimental signal. 


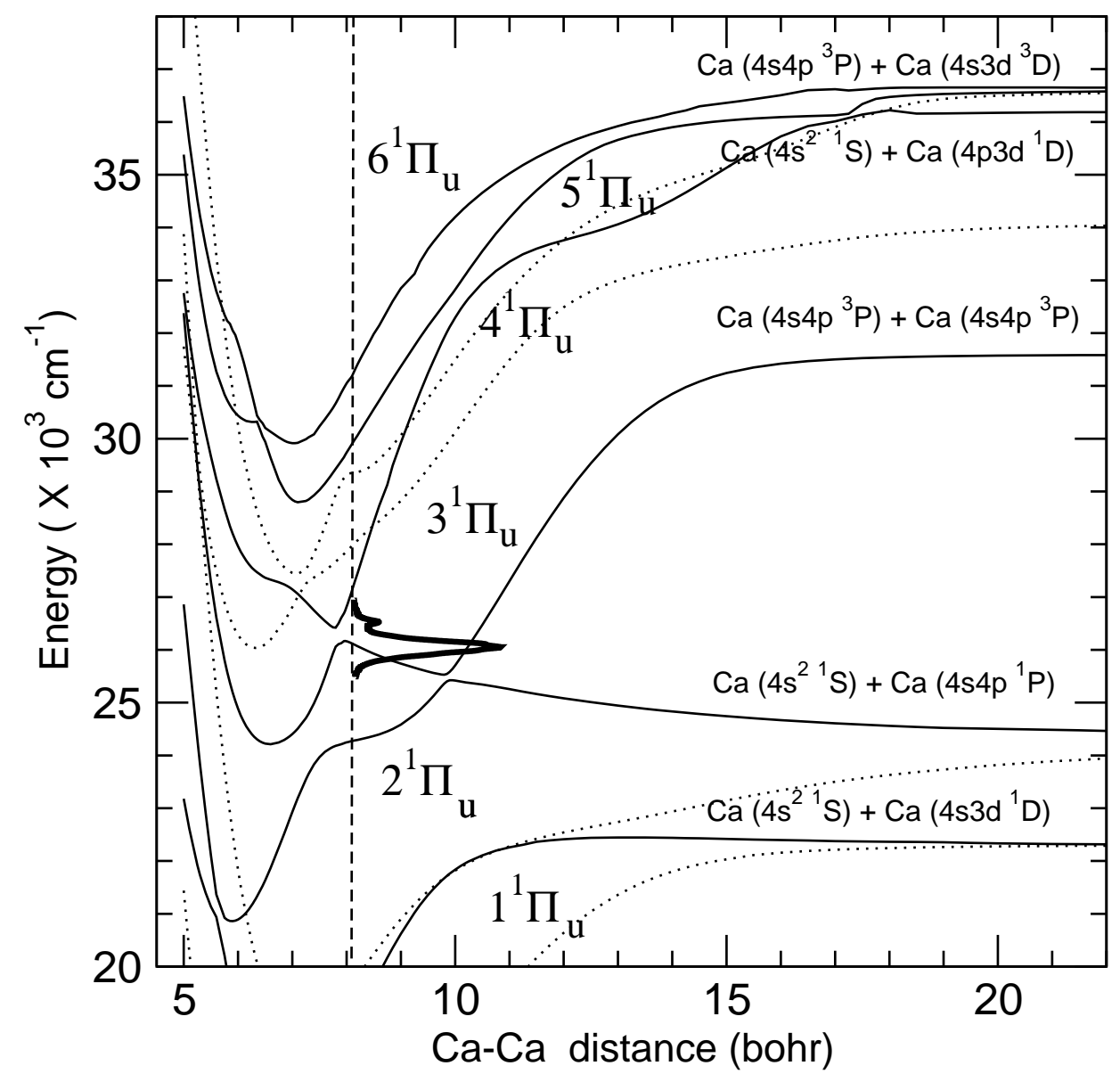

Figure 3: Ab initio potential curves of ${ }^{1} \Pi_{\mathrm{u}}$ symmetry correlating to the asymptotes labeled in the figure. The four lowest energy ${ }^{1} \Sigma_{\mathrm{u}}$ states are indicated by dotted lines. The zero energy is taken at the bottom of the ground state well of $\mathrm{Ca}_{2}$. The vertical dashed line shows the calculated equilibrium distance of the ground state. The theoretical absorption spectrum is represented in thick solid lines, with the zero-intensity corresponding to the above mentioned dashed line. 


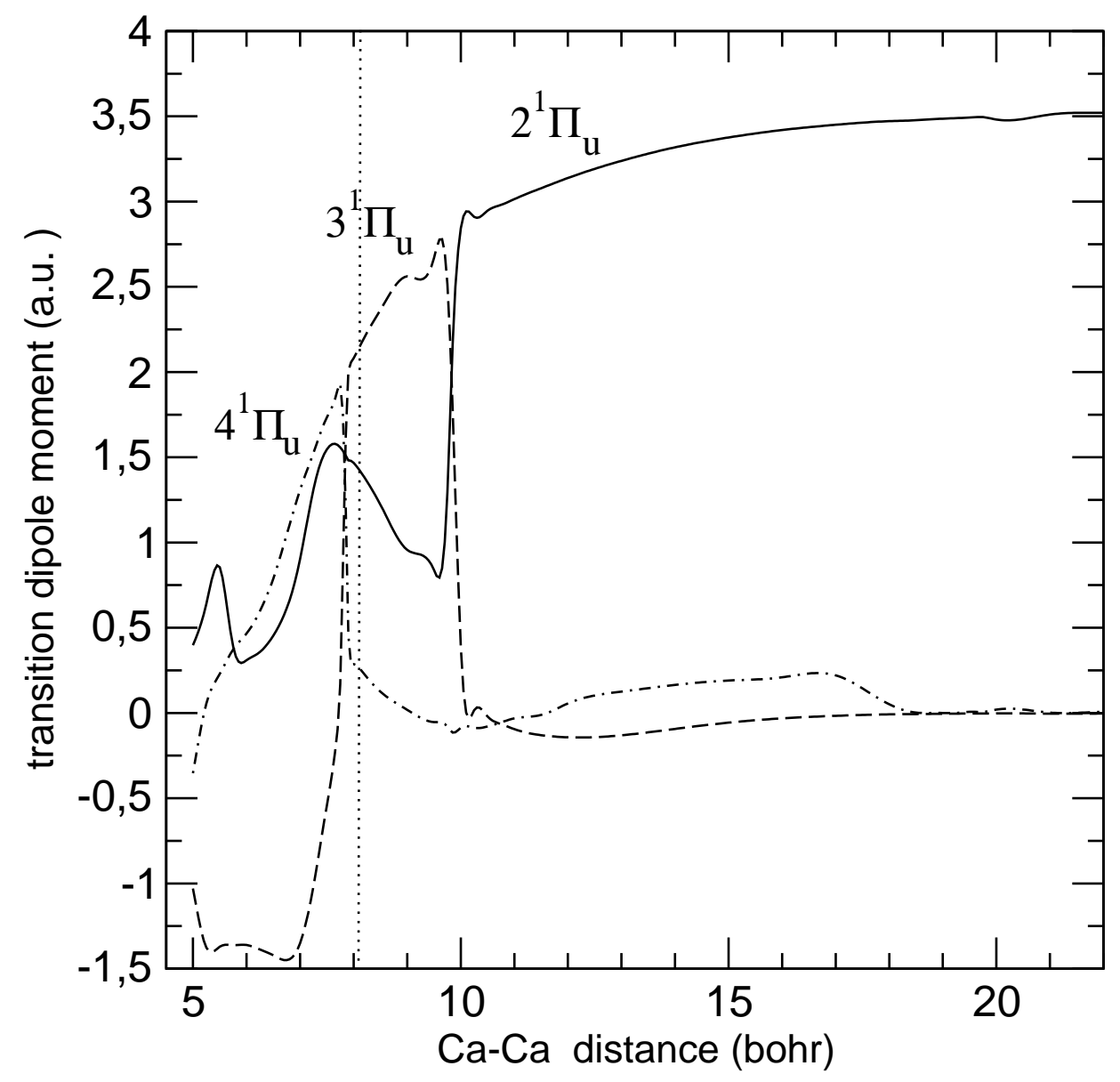

Figure 4: Electronic transition dipole moment of the $2^{1} \Pi_{u}, 3^{1} \Pi_{u}$ and $4^{1} \Pi_{u}$ states (respectively with solid, dashed and dashed-dot lines) from the electronic ground state of $\mathrm{Ca}_{2}$. The vertical dotted line shows the calculated equilibrium distance of the electronic ground state. 


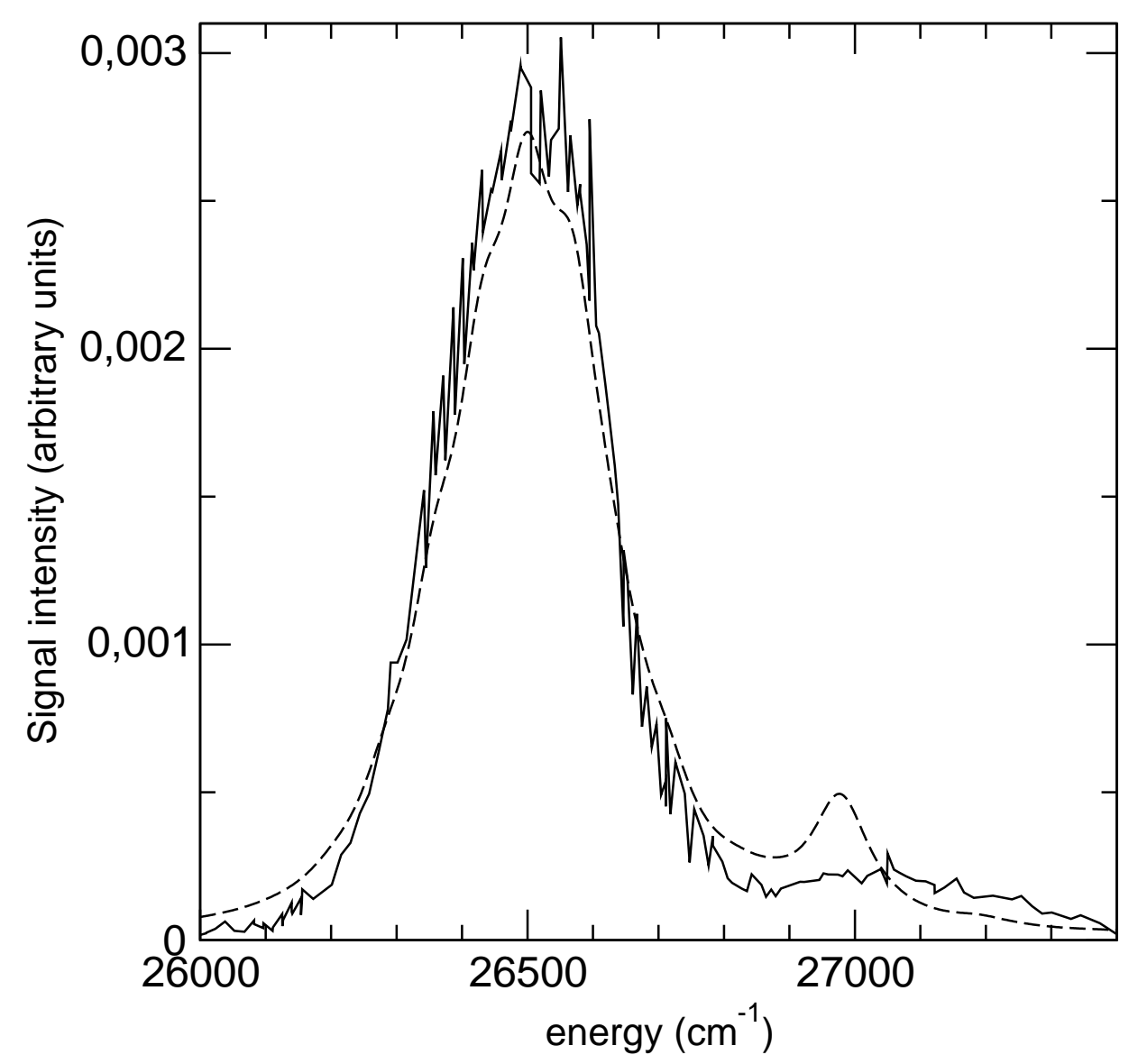

Figure 5: Excitation spectrum of the action spectrum of process (1) in the helium experiment (solid line) compared to the theoretical absorption spectrum (dashed line) of $\mathrm{Ca}_{2}$. The two spectra are normalized with respect to their intensity. The theoretical spectrum is shifted $450 \mathrm{~cm}^{-1}$ to the blue. 